\title{
A METHOD FOR SHOCK CALCULATION
}

\author{
V. F.Kuropatenko ${ }^{1}$, v.f.kuropatenko@rambler.ru,
}

M. N. Yakimova ${ }^{1}$, yakimovamn@yandex.ru.

${ }^{1}$ Russian Federal Nuclear Center E. I. Zababakhin All-Russian Scientific Research Institute of Technical Physics, Snezhinsk, Russian Federation.

The mass, momentum and energy conservation laws allow shock and rarefaction waves to be present in the solution of continuum mechanics problems. When these problems are solved with homogeneous difference techniques, the strong shock surface is represented by a layer of a finite width within which the quantities vary continuously from a state before the shock front to a state behind it. These states are related by the strong shock conditions. Since they lie on the Hugoniot, there must exist a mechanism which maintains energy dissipation in the shock layer. One of these mechanisms is a method by Kuropatenko which uses the difference equations applicable for strong shocks. The method can be implemented in different difference schemes. The paper presents one of them, describes its basic properties, and provides results of some calculations.

Keywords: conservation laws, energy dissipation, difference scheme, shock, rarefaction.

\section{Introduction}

In $1 \mathrm{D}$, the laws of mass, momentum and energy conservation for ideal continuum with no heat conduction in Lagrangian coordinates for the case of plane symmetry read as

$$
\begin{gathered}
\frac{\partial V}{\partial t}-\frac{\partial U}{\partial M}=0 \\
\frac{\partial U}{\partial t}+\frac{\partial P}{\partial M}=0 \\
\frac{\partial \varepsilon}{\partial t}+\frac{\partial(P U)}{\partial M}=0
\end{gathered}
$$

where $t$ is time, $M$ is mass coordinate, $U$ is material velocity, $V$ is specific volume, $P$ is pressure, $\varepsilon=E+0.5 U^{2}$ is specific total energy, and $E$ is specific internal energy.

The system of equations (1)-(3) is closed by the equation of state

$$
P=F(V, E)
$$

and the equation of trajectory for a particle with coordinate $M$

$$
\left(\frac{\partial x}{\partial t}\right)_{M}-U=0
$$

where $x$ is the Eulerian coordinate of the particle.

Since equations (1)-(3) are not linear, they allow strong and weak discontinuities to be present in continuum mechanics problems. Material states before and behind the shock front are related by the system of non-linear algebraic equations (Hugoniot conditions)

$$
\begin{gathered}
P_{1}-P_{0}-W\left(U_{1}-U_{0}\right)=0 \\
U_{1}-U_{0}+W\left(V_{1}-V_{0}\right)=0 \\
\left(E_{1}+0.5 U_{1}^{2}-E_{0}-0.5 U_{0}^{2}\right) W-\left(P_{1} U_{1}-P_{0} U_{0}\right)=0 .
\end{gathered}
$$


Here $W$ is strong shock velocity, and the subscript 0 corresponds to the state before the front and the subscript 1 corresponds to the state behind the front. Entropy jumps on the strong shock surface. This is the key difference between the shock waves and the waves where the quantities vary continuously.

When homogeneous difference methods are used for hydrodynamics, the strong shock surface is represented by a layer of a finite width [1,2]. The strong shock smears [3]. In the layer, the quantities continuously vary from a state before the shock front to a state behind it. Since entropy jumps across the shock, there arises the question about the mechanism of energy dissipation in the shock layer. In the literature we can find four basically different mechanisms of energy dissipation [4, 7]. Consider a difference scheme based on the method proposed in [7].

\section{Basic equations}

The difference equations are written for a staggered mesh (Fig. 1). Velocities and coordinates are updated on the boundaries of intervals and the thermodynamic quantities are updated in their centers.

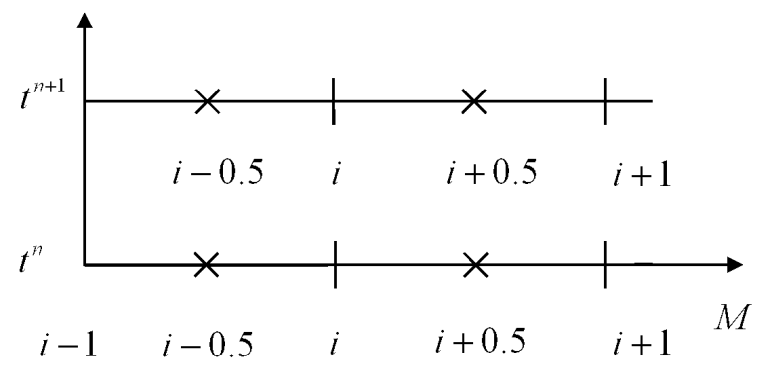

Fig. 1. Staggered mesh

All intervals are divided into those where material is compressed $\left(U_{i}^{n}-U_{i-1}^{n}<0\right)$ and those where material expands $\left(U_{i}^{n}-U_{i-1}^{n} \geqslant 0\right)$. For the intervals of the first type, equations (1)-(5) are approximated by the difference equations

$$
\begin{gathered}
x_{i}^{n+1}=x_{i}^{n}+\tau U_{i}^{n}, V_{i-0.5}^{n+1}=\frac{x_{i}^{n+1}-x_{i-1}^{n+1}}{\Delta M_{i-0.5}^{n}} \\
U_{i}^{n+1}=U_{i}^{n}-\frac{2 \tau}{\Delta M_{i+0.5}^{n}+\Delta M_{i-0.5}^{n}}\left(\bar{P}_{i+0.5}^{n}-\bar{P}_{i-0.5}^{n}\right), \\
\varepsilon_{i-0.5}^{n+1}=E_{i-0.5}^{n}+0.5\left(U_{i-0.5}^{* n}\right)^{2}-\frac{\tau}{\Delta M_{i-0.5}^{n}}\left(P_{i}^{*} U_{i}^{n}-P_{i-1}^{*} U_{i-1}^{n}\right), \\
P_{i-0.5}^{n+1}=F\left(V_{i-0.5}^{n+1}, E_{i-0.5}^{n+1}\right),
\end{gathered}
$$

where $\Delta M_{i-0.5}^{n}=M_{i}^{n}-M_{i-1}^{n}$.

In equations (9)-(12) we use the auxiliary quantities $\bar{P}^{n}, P^{*}$ and $U^{*}$. The quantity $\bar{P}^{n}$ is derived from Hugoniot conditions (6)-(8) and $\Delta U^{n}$ which is known. The method to derive $P^{*}$ ad $U^{*}$ will be described below. 
For the intervals of the second type, the difference equations are written as

$$
\begin{gathered}
x_{i}^{n+1}=x_{i}^{n}+\tau U_{i}^{n}, V_{i-0.5}^{n+1}=\frac{x_{i}^{n+1}-x_{i-1}^{n+1}}{\Delta M_{i-0.5}^{n}} \\
U_{i}^{n+1}=U_{i}^{n}-\frac{2 \tau}{\Delta M_{i+0.5}^{n}+\Delta M_{i-0.5}^{n}}\left(\bar{P}_{i+0.5}^{n}-\bar{P}_{i-0.5}^{n}\right), \\
E_{i-0.5}^{n+1}=E_{i-0.5}^{n}-\int_{V^{n}} P_{i-0.5}\left(V_{i-0.5}, E_{i-0.5}\right) d V, \\
P_{i-0.5}^{n+1}=F\left(V_{i-0.5}^{n+1}, E_{i-0.5}^{n+1}\right),
\end{gathered}
$$

where $\bar{P}_{i-0.5}^{n}=P_{i-0.5}^{n}-a_{i-0.5}^{n} \varkappa_{i-0.5}\left(U_{i}^{n}-U_{i-1}^{n}\right), a_{i-0.5}^{n}=\rho_{i-0.5}^{n} c_{i-0.5}^{n}, c=\sqrt{\left(\frac{\partial P}{\partial \rho}\right)_{S}}$ is adiabatic sound velocity, and $\varkappa_{i-0.5}=\frac{\tau a_{i-0.5}^{n}}{\Delta M_{i-0.5}^{n}}$ is local Courant number. Internal energy at step $\mathrm{n}+1$ is calculated by integrating (15) and (16) along the isentrope which ensures any predefined accuracy in the determination of entropy.

\section{Determination of auxiliary quantities}

The quantities $P^{*}$ and $U^{*}$ are defined on the following mesh (Fig. 2).

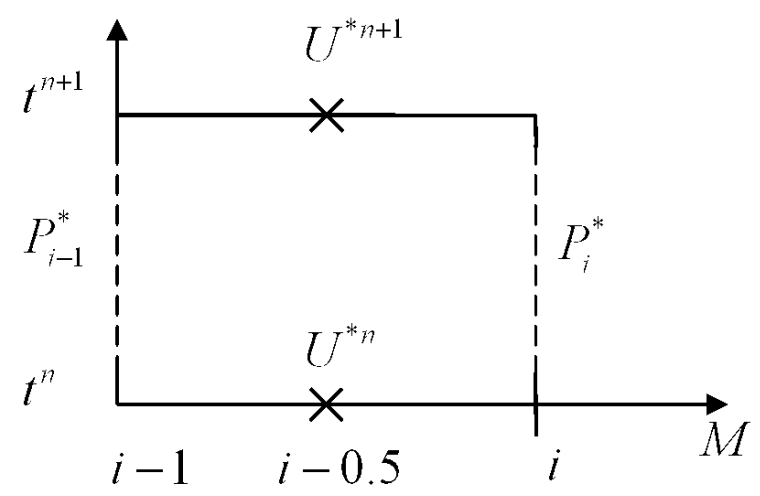

Fig. 2. Mesh for auxiliary functions

The values of $P_{i}^{*}$ are defined by solutions in the intervals on the right and left from point $i$ in accord with Table 1 .

The quantity $U_{i-0.5}^{* n}$ for the intervals of the first type is defined with respect to the shock direction.

If $P_{i+0.5}^{n}<P_{i-1.5}^{n}$, the wave moves right $W_{i-0.5}^{n}>0$ and $U_{i-0.5}^{* n}=U_{i}^{n}$.

If $P_{i+0.5}^{n} \geqslant P_{i-1.5}^{n}$, the wave moves left $W_{i-0.5}^{n}<0$ and $U_{i-0.5}^{* n}=U_{i-1}^{n}$.

In the intervals of the second type $W_{i-0.5}^{n}=0$ and $U_{i-0.5}^{* n}$ is calculated as the half-sum of boundary velocities: $U_{i-0.5}^{* n}=0.5\left(U_{i-1}^{n}+U_{i}^{n}\right)$. 
Table 1

The values of $P_{i}^{*}$

\begin{tabular}{|l|c|c|c|}
\hline$N$ & $\begin{array}{c}\text { Interval } i-0.5 \\
\omega_{L}=W_{i-0.5}^{n}\left(U_{i}^{n}-U_{i-1}^{n}\right)\end{array}$ & $\begin{array}{c}\text { Interval } i+0.5 \\
\omega_{R}=W_{i+0.5}^{n}\left(U_{i+1}^{n}-U_{i}^{n}\right)\end{array}$ & \multicolumn{1}{c|}{$P_{i}^{*}$} \\
\hline 1 & $\omega_{L}=0$ & $\omega_{R}=0$ & $P_{i}^{*}=0.5\left(\bar{P}_{i-0.5}^{n}+\bar{P}_{i+0.5}^{n}\right)$ \\
\hline 2 & $\omega_{L}=0$ & $\omega_{R}<0$ & $P_{i}^{*}=0.5\left(\bar{P}_{i-0.5}^{n}+P_{i+0.5}^{n}\right)$ \\
\hline 3 & $\omega_{L}=0$ & $\omega_{R}>0$ & $P_{i}^{*}=\bar{P}_{i+0.5}^{n}$ \\
\hline 4 & $\omega_{L}>0$ & $\omega_{R}=0$ & $P_{i}^{*}=0.5\left(P_{i-0.5}^{n}+\bar{P}_{i+0.5}^{n}\right)$ \\
\hline 5 & $\omega_{L}>0$ & $\omega_{R}<0$ & $P_{i}^{*}=0.5\left(P_{i-0.5}^{n}+P_{i+0.5}^{n}\right)$ \\
\hline 6 & $\omega_{L}>0$ & $\omega_{R}>0$ & $P_{i}^{*}=\bar{P}_{i+0.5}^{n}$ \\
\hline 7 & $\omega_{L}<0$ & $\omega_{R}=0$ & $P_{i}^{*}=\bar{P}_{i-0.5}^{n}$ \\
\hline 8 & $\omega_{L}<0$ & $\omega_{R}<0$ & $P_{i}^{*}=\bar{P}_{i-0.5}^{n}$ \\
\hline 9 & $\omega_{L}<0$ & $\omega_{R}>0$ & $P_{i}^{*}=0.5\left(\bar{P}_{i-0.5}^{n}+\bar{P}_{i+0.5}^{n}\right)$ \\
\hline
\end{tabular}

The quantity $U_{i-0.5}^{* n+1}$ which is needed for the determination of internal energy $E_{i-0.5}^{n+1}=\varepsilon_{i-0.5}^{n+1}-0.5\left(U_{i-0.5}^{* n+1}\right)^{2}$, is calculated from the equation

$$
U_{i-0.5}^{* n+1}=U_{i-0.5}^{* n}-\frac{\tau}{\Delta M_{i-0.5}}\left(P_{i}^{*}-P_{i-1}^{*}\right) .
$$

\section{Basic properties of the difference scheme}

\subsection{Approximation errors}

According to [8], the differential conservation laws are written as

$$
\begin{gathered}
\frac{\partial x}{\partial t}-U=\omega_{4}, \frac{\partial x}{\partial M}-V=\omega_{5} \\
\frac{\partial U}{\partial t}+\frac{\partial P}{\partial M}=\omega_{2} \\
\frac{\partial \varepsilon}{\partial t}+\frac{\partial(P U)}{\partial M}=\omega_{3}
\end{gathered}
$$

where the approximation errors $\omega_{4}, \omega_{5}, \omega_{2}$ and $\omega_{3}$ for an acoustically approximated shock $(W=a)$ are as follows

$$
\begin{gathered}
\omega_{4}=-\frac{\tau}{2} \dot{U}+\frac{\tau^{2}}{12} \ddot{U}+o\left(\tau^{3}\right), \omega_{5}=-V^{\prime \prime} \frac{h^{2}}{24}+o\left(h^{3}\right), \\
\omega_{2}=-\frac{\tau}{2} \ddot{U}-\frac{\tau^{2}}{6} \dddot{U}+a h U^{\prime \prime}-\frac{h^{2}}{24} P^{\prime \prime \prime}+o\left(\tau^{3}, h^{3}\right), \\
\omega_{3}=-\frac{\tau}{2} \ddot{\varepsilon}-\frac{\tau^{2}}{6} \dddot{\varepsilon}-h\left(\frac{1}{2} P^{\prime} U^{\prime}+\frac{1}{2} P^{\prime \prime} U-a\left(U^{\prime}\right)^{2}-a U U^{\prime \prime}\right)+o\left(\tau^{3}, h^{2}\right) .
\end{gathered}
$$


For the rarefaction wave, they are

$$
\begin{aligned}
& \omega_{4}=-\frac{\tau}{2} \dot{U}+\frac{\tau^{2}}{12} \ddot{U}+o\left(\tau^{3}\right), \omega_{5}=-V^{\prime \prime} \frac{h^{2}}{24}+o\left(h^{3}\right), \\
& \omega_{2}=-\tau\left(\frac{\ddot{U}}{2}-a^{2} U^{\prime \prime}\right)-\frac{\tau^{2}}{6} \dddot{U}-\frac{h^{2}}{24} P^{\prime \prime \prime}+o\left(\tau^{3}, h^{3}\right) .
\end{aligned}
$$

The entropy production equation for rarefaction is $T\left(\frac{\partial S}{\partial t}\right)_{M}=0$.

Thus the approximation errors are first order infinitesimals in $\tau$ and $\Delta M$ on the shock wave, and first order infinitesimal in time and second order infinitesimal in the Lagrangian coordinate on the rarefaction wave.

\subsection{Stability condition}

Theorem 1. The difference scheme under consideration is conditionally stable.

Proof.

We use harmonics. For finding the stability condition, we write the difference scheme in the acoustic case

$$
\frac{U_{i}^{n+1}-U_{i}^{n}}{\tau}+\frac{\bar{P}_{i+0.5}^{n}-\bar{P}_{i-0.5}^{n}}{h}=0 ; \frac{P_{i+0.5}^{n+1}-P_{i+0.5}^{n}}{\tau}+a^{2} \frac{U_{i+1}^{n}-U_{i}^{n}}{h}=0 .
$$

Substituting

$$
\delta P_{i+0.5}^{n}=\delta P_{0} e^{\alpha t^{n}+i \beta m_{i+0.5}}, \delta U_{i}^{n}=\delta U_{0} e^{\alpha t^{n}+i \beta m_{i}}, e^{\alpha \tau}=\lambda, e^{i \beta \frac{h}{2}}=\xi
$$

yields

$$
\begin{gathered}
U_{0}\left(\lambda-1-\frac{\tau a}{h}\left(\xi^{2}-2+\xi^{-2}\right)\right)+\frac{\tau}{h} P_{0}\left(\xi-\xi^{-1}\right)=0, \\
\frac{\tau a^{2}}{h} U_{0}\left(\xi-\xi^{-1}\right)+P_{0}(\lambda-1)=0 .
\end{gathered}
$$

The determinant of this system is

$$
(\lambda-1)^{2}-\varkappa(\lambda-1)\left(\xi^{2}-2+\xi^{-2}\right)-\varkappa^{2}\left(\xi^{2}-2+\xi^{-2}\right)=0
$$

where $\varkappa=\frac{\tau a}{h}$. We solve this equation and obtain that $|\lambda| \leqslant 1$ at $\frac{\tau a}{h} \leqslant 1$.

\subsection{Monotony condition}

Let a shock move to the right from point i $(\mathrm{W}>0)$. On the shock, equations (17) take the form

$$
U_{i+0.5}^{n+1}=U_{i+0.5}^{n}-\frac{\tau}{\Delta M_{i+0.5}}\left(P_{i+1}^{*}-P_{i}^{*}\right), P_{i+0.5}^{n+1}=P_{i+0.5}^{n}-\frac{\tau a^{2}}{h}\left(U_{i+1}^{n}-U_{i}^{n}\right) .
$$


Velocity in interval $(\mathrm{i}+0.5)$ is constant and equal to $U_{i+1}^{n}$. All quantities in interval $(\mathrm{i}+0.5)$ are before the shock front. With $P_{i}^{*}=P_{i+0.5}^{n}-W_{i+0.5}^{n}\left(U_{i+1}^{n}-U_{i}^{n}\right)$ and $P_{i+1}^{*}=P_{i+1.5}^{n}-W_{i+1.5}^{n}\left(U_{i+2}^{n}-U_{i+1}^{n}\right)$ equation (18) takes the form

$$
\begin{gathered}
U_{i+0.5}^{n+1}=U_{i+0.5}^{n}-\frac{\tau}{h}\left(\left(P_{i+1.5}^{n}-P_{i+0.5}^{n}\right)-a\left(U_{i+1.5}^{n}-2 U_{i+0.5}^{n}+U_{i-0.5}^{n}\right)\right), \\
P_{i+0.5}^{n+1}=P_{i+0.5}^{n}-\frac{\tau a^{2}}{h}\left(U_{i+0.5}^{n}-U_{i-0.5}^{n}\right) .
\end{gathered}
$$

With the Riemann invariants $\alpha=P+a U, \beta=P-a U$, we convert (19)-(20) to

$$
\begin{aligned}
\alpha_{i+0.5}^{n+1}-\beta_{i+0.5}^{n+1} & =\alpha_{i+0.5}^{n}-\beta_{i+0.5}^{n}-\varkappa\left(\alpha_{i+1.5}^{n}+\beta_{i+1.5}^{n}-\alpha_{i+0.5}^{n}-\beta_{i+0.5}^{n}\right)+ \\
& +\varkappa\left(\alpha_{i+1.5}^{n}-\beta_{i+1.5}^{n}-2\left(\alpha_{i+0.5}^{n}-\beta_{i+0.5}^{n}\right)+\alpha_{i-0.5}^{n}-\beta_{i-0.5}^{n}\right), \\
\alpha_{i+0.5}^{n+1}+\beta_{i+0.5}^{n+1} & =\alpha_{i+0.5}^{n}+\beta_{i+0.5}^{n}-\varkappa\left(\alpha_{i+0.5}^{n}-\beta_{i+0.5}^{n}-\alpha_{i-0.5}^{n}+\beta_{i-0.5}^{n}\right) .
\end{aligned}
$$

Summing these equation gives

$$
2 \alpha_{i+0.5}^{n+1}=2(1-\varkappa) \alpha_{i+0.5}^{n}+2 \varkappa \alpha_{i-0.5}^{n}+4 \varkappa \beta_{i+0.5}^{n}-2 \varkappa \beta_{i+1.5}^{n}-2 \varkappa \beta_{i-0.5}^{n} .
$$

Taking into account that for a right moving wave, $\beta=$ const, we obtain

$$
\alpha_{i+0.5}^{n+1}=(1-\varkappa) \alpha_{i+0.5}^{n}+\varkappa \alpha_{i-0.5}^{n}
$$

By Godunov's theorem, a scheme is monotone if coefficients in (21) are non-negative. This is true at $\varkappa \leqslant 1$.

Do similar manipulations for rarefaction. In this case, equations (17) with $\bar{P}_{i-0.5}^{n}=P_{i-0.5}^{n}-a_{i-0.5}^{n} \varkappa\left(U_{i}^{n}-U_{i-1}^{n}\right)$ take the form

$$
\begin{gathered}
U_{i}^{n+1}=U_{i}^{n}-\frac{\tau}{h}\left(P_{i+0.5}^{n}-P_{i-0.5}^{n}\right)+\varkappa^{2}\left(U_{i+1}^{n}-2 U_{i}^{n}+U_{i-1}^{n}\right) \\
P_{i+0.5}^{n+1}=P_{i+0.5}^{n}-a \varkappa\left(U_{i+1}^{n}-U_{i}^{n}\right) .
\end{gathered}
$$

Substituting yields

$$
\begin{aligned}
\alpha_{i}^{n+1}-\beta_{i}^{n+1}= & \alpha_{i}^{n}-\beta_{i}^{n}-\varkappa\left(\alpha_{i+0.5}^{n}+\beta_{i+0.5}^{n}-\alpha_{i-0.5}^{n}-\beta_{i-0.5}^{n}\right)+\varkappa^{2}\left(\alpha_{i+1}^{n}-\beta_{i+1}^{n}-2\left(\alpha_{i}^{n}-\beta_{i}^{n}\right)+\alpha_{i-1}^{n}-\beta_{i-1}^{n}\right), \\
& \alpha_{i+0.5}^{n+1}+\beta_{i+0.5}^{n+1}=\alpha_{i+0.5}^{n}+\beta_{i+0.5}^{n}-\varkappa\left(\alpha_{i+1}^{n}-\beta_{i+1}^{n}-\alpha_{i}^{n}+\beta_{i}^{n}\right) .
\end{aligned}
$$

Summing with account for $\beta=$ const gives

$$
\alpha_{i}^{n+1}=0.5 \varkappa^{2} \alpha_{i+1}^{n}-\varkappa \alpha_{i+0.5}^{n}+\left(1-\varkappa^{2}\right) \alpha_{i}^{n}+\varkappa \alpha_{i-0.5}^{n}+0.5 \varkappa^{2} \alpha_{i-1}^{n} .
$$

One of the coefficients is negative and therefore the scheme is non-monotone on rarefaction waves. 


\section{Generalization to spherical and cylindrical cases}

In 1D, hydrodynamic equations for ideal medium read as

$$
\begin{gathered}
\left(\frac{\partial r}{\partial t}\right)_{M}=U, V=\frac{\partial r^{\alpha}}{\partial M}, \\
\frac{\partial U}{\partial t}+\alpha r^{\alpha-1} \frac{\partial P}{\partial M}=0 \\
\frac{\partial \varepsilon}{\partial t}+\frac{\partial\left(\alpha r^{\alpha-1} U P\right)}{\partial M}=0,
\end{gathered}
$$

where $\alpha$ is dimensionality ( $\alpha=1$ for plane, $\alpha=2$ for cylinder, and $\alpha=3$ for sphere), and the Lagrangian coordinate $M$ reads as

$$
M=\int_{0}^{r} \rho r^{\alpha-1} d r .
$$

Equations (24)-(26) in finite differences are written as

$$
\begin{gathered}
r_{i}^{n+1}=r_{i}^{n}+\tau U_{i}^{n}, V_{i-0.5}^{n+1}=\frac{\left(r_{i}^{n+1}\right)^{\alpha}-\left(r_{i-1}^{n+1}\right)^{\alpha}}{\Delta M_{i-0.5}^{n}}, \\
U_{i}^{n+1}=U_{i}^{n}-\alpha\left(r_{i}^{n}\right)^{\alpha-1} \frac{\tau}{M_{i+}^{n}+\Delta M_{i-}^{n}}\left(P_{i+0.5}^{n}-P_{i-0.5}^{n}\right), \\
\varepsilon_{i-0.5}^{n+1}=E_{i-0.5}^{n}+0.5\left(U_{i-0.5}^{* n}\right)^{2}-\alpha \frac{\tau}{\Delta M_{i-0.5}^{n}}\left(\left(r_{i}^{n}\right)^{\alpha-1} P_{i}^{*} U_{i}^{n}-\left(r_{i-1}^{n}\right)^{\alpha-1} P_{i-1}^{*} U_{i-1}^{n}\right),
\end{gathered}
$$

where $M_{i-}^{n}=\rho_{i-0.5}^{n}\left(\left(r_{i}^{n}\right)^{\alpha}-\left(r_{i-0.5}^{n}\right)^{\alpha}\right)$ is the mass of a half of interval $\mathrm{i}$ and $M_{i+}^{n}=\rho_{i+0.5}^{n}\left(\left(r_{i+0.5}^{n}\right)^{\alpha}-\left(r_{i}^{n}\right)^{\alpha}\right)$ is the mass of a half of interval $\mathrm{i}+1$ (see Fig. 3 ). For plane with $\alpha=1$, these masses equal the halves of the masses of the intervals.

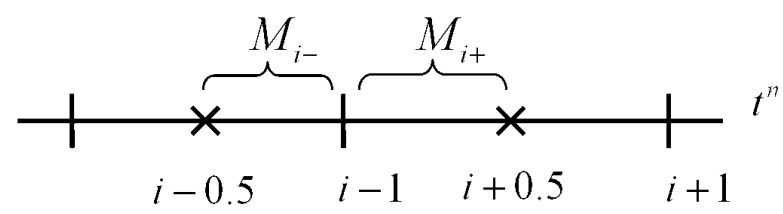

Fig. 3. Mesh

The auxiliary quantity $U_{i-0.5}^{* n+1}$ is calculated from the equation of motion

$$
U_{i-0.5}^{* n+1}=U_{i-0.5}^{* n}-\alpha\left(r_{i-0.5}^{n}\right)^{\alpha-1} \frac{\tau}{\Delta M_{i-0.5}^{n}}\left(P_{i}^{*}-P_{i-1}^{*}\right)
$$

\section{Verification}

The figures below compare results for some problems from [9] which were calculated with the proposed scheme, and their analytical solutions. All calculations were done with Courant number 0.5. 
Example 1. Propagation of a stationary shock in ideal gas.

At the initial time $t=0$, ideal gas, $\gamma=4 / 3$, is in the region $0 \leqslant x \leqslant 14$.

Initial conditions: $\rho_{0}=1, E_{0}=0, P_{0}=0, U_{0}=0$.

Boundary conditions: $U_{L}=3, U_{R}=0$. A uniform mesh of 100 intervals was used.
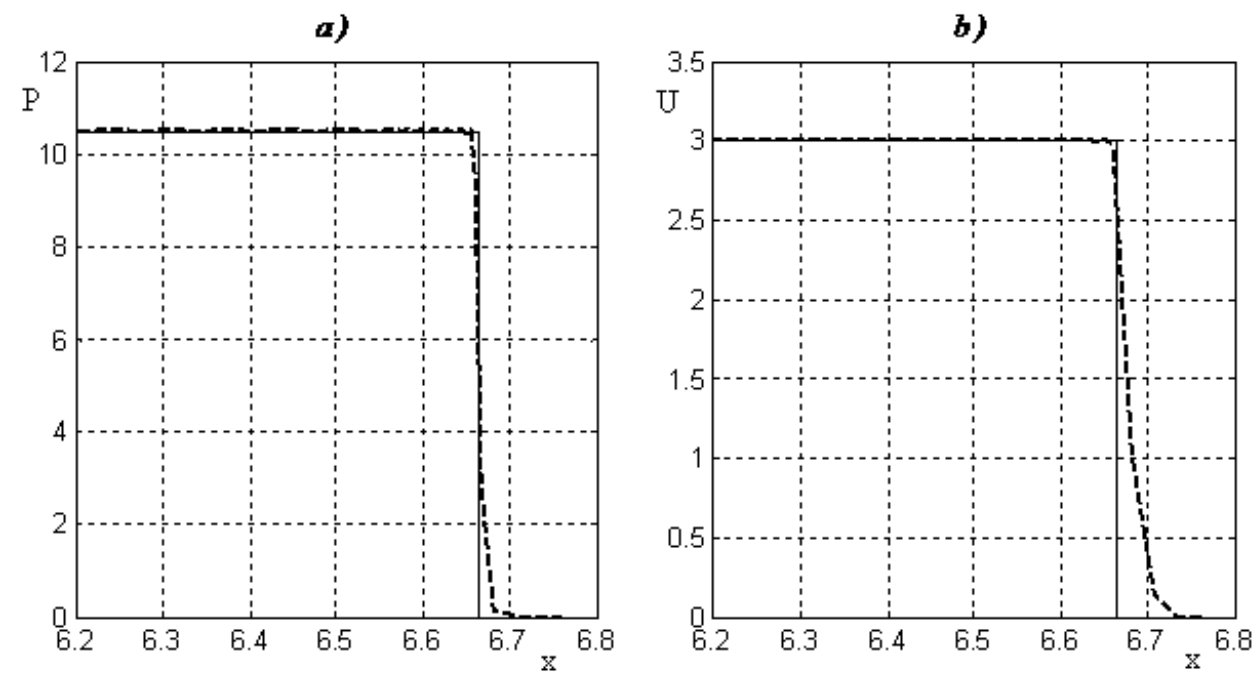

Fig. 4. Pressure and velocity in the stationary shock

Figures $4 \mathrm{a}$ and $4 \mathrm{~b}$ show $P(x)$ and $U(x)$ profiles at $t=1.904$. The solid line shows the analytical solution and the dashed one shows calculation with the proposed method.

Example 2. Propagation of a rarefaction wave in ideal gas.

At the initial time $t=0$, ideal gas, $\gamma=2$, is in the region $0 \leqslant x \leqslant 14$.

Initial conditions: $\rho_{0}=4.5, E_{0}=1.125, P_{0}=5.0625, U_{0}=0$.

Boundary conditions: $U_{L}=-1, U_{R}=0$. A uniform mesh of 100 intervals was used. Figures $5 \mathrm{a}$ and $5 \mathrm{~b}$ show $P(x)$ and $U(x)$ profiles at $t=4.043$. The solid line shows analytics
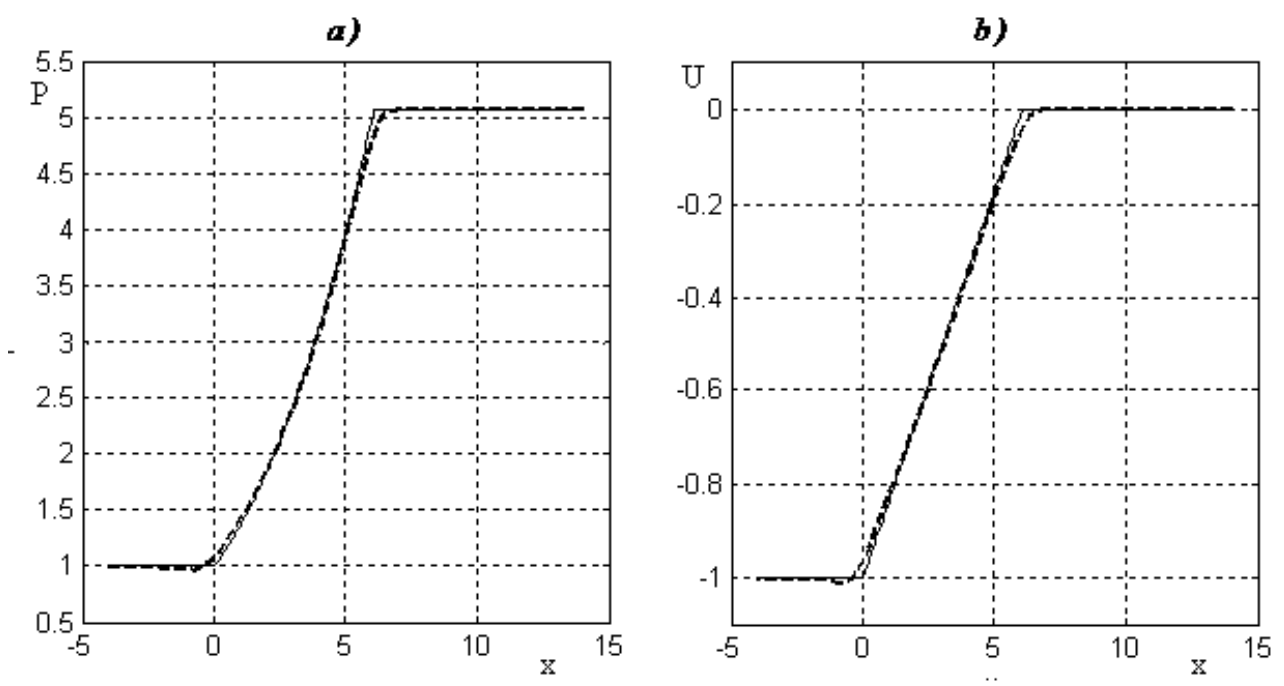

Fig. 5. Pressure and velocity in the rarefaction wave 
and the dashed one shows calculation with the proposed method.

Example 3. A spherically symmetric shock converging to the center.

At the initial time $t=0$, the cold ideal gas, $\gamma=5 / 3$, is at rest in the region $0 \leqslant r \leqslant 1$.

Initial conditions: $\rho_{0}=1, E_{0}=0, P_{0}=0, U_{0}=0$.

The condition on the boundary of the gas sphere is taken from the analytical solution [9] as a table of $\mathrm{U}$ versus $\mathrm{t}$ (Table 2).

Table 2

\begin{tabular}{|c|c|c|c|c|c|}
\hline$N$ & $t$ & $U_{R}$ & $N$ & $t$ & $U_{R}$ \\
\hline 1 & 0 & -1 & 9 & 0.328273 & -1.04392 \\
\hline 2 & 0.02 & -1.004474 & 10 & 0.36 & -1.0395 \\
\hline 3 & 0.102828 & -1.02181 & 11 & 0.39144 & -1.03309 \\
\hline 4 & 0.16 & -1.0323 & 12 & 0.42 & -1.0227 \\
\hline 5 & 0.20701 & -1.0391 & 13 & 0.438247 & -1.01479 \\
\hline 6 & 0.241516 & -1.04277 & 14 & 0.475136 & -0.991499 \\
\hline 7 & 0.286642 & -1.04513 & 15 & 0.50547 & -0.964469 \\
\hline 8 & 0.31 & -1.045 & 16 & 0.540474 & -0.92164 \\
\hline
\end{tabular}

A uniform mesh of 200 intervals was used.

a)

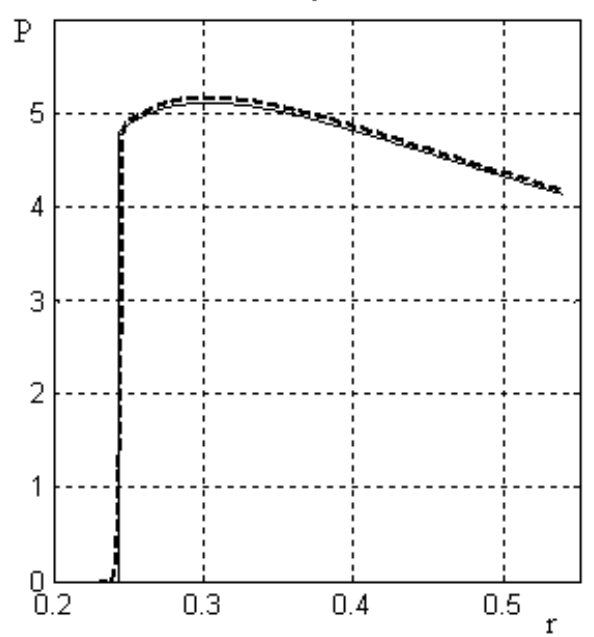

b)

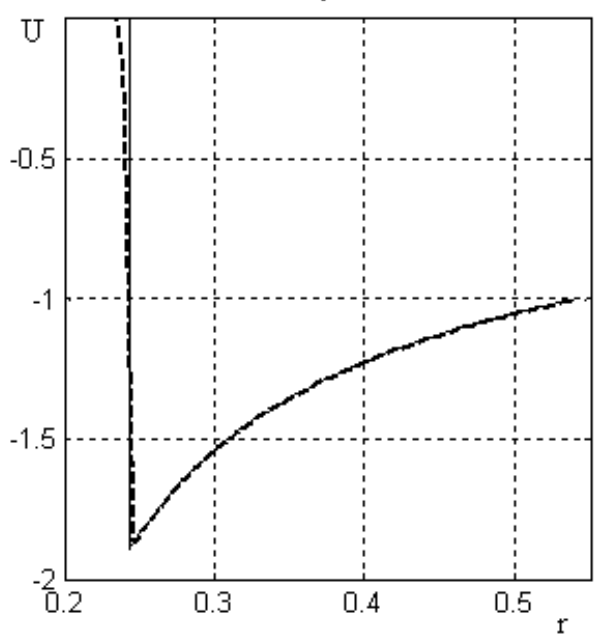

Fig. 6. Pressure and velocity in the spherically symmetric shock

Figures $6 \mathrm{a}$ and $6 \mathrm{~b}$ show $P(r)$ and $U(r)$ profiles at $t=0.45$. The solid line shows analytics from [9] and the dashed one shows calculation with the proposed method.

Example 4. Shock interaction with interface at $\frac{\rho_{R}}{\rho_{L}}=0.5$ in ideal gas.

At the initial time $t=0$, gas with the initial state $\rho_{0}=1, E_{0}=0, P_{0}=0, U_{0}=0$ is in the region $0 \leqslant r \leqslant 14$, and gas with the initial state $\rho_{0}=0.5, E_{0}=0, P_{0}=0, U_{0}=0$ is in the region $14 \leqslant r \leqslant 28$. In both the regions, the equation of state is ideal gas with $\gamma=4 / 3$. 
Boundary conditions: $U_{L}=3, U_{R}=0$.

A uniform mesh was used with 200 intervals in the first region and 100 intervals in the second one.
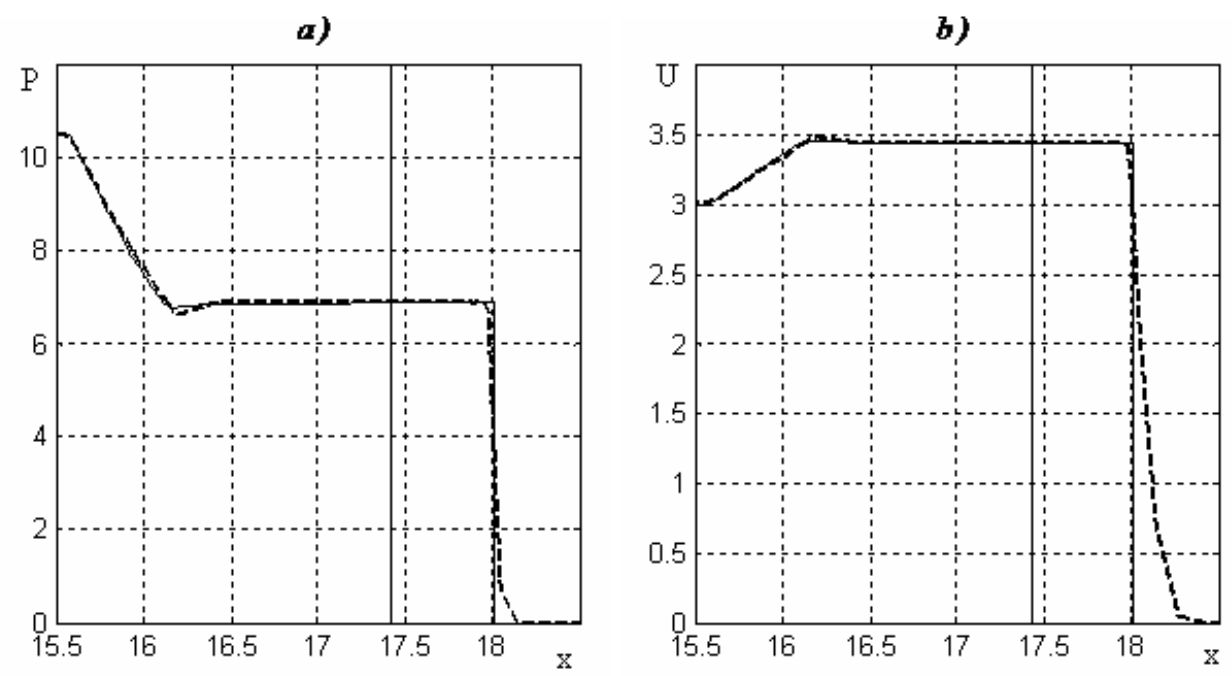

Fig. 7. Pressure and velocity in the shock interaction

Figures 7a and 7b show $P(x)$ and $U(x)$ profiles at $t=5$. The solid line shows calculation by the VOLNA code [9] with shock capturing, and the dashed one shows calculation with the proposed method. The vertical line at $\mathrm{x}=17.44$ shows the position of the interface at that time.

This work was assumed by RFBR, project 13-01-00072.

\section{References}

1. Rozhdestvensky B.L., Yanenko N.N. [Systems of Quasi-linear Equations and Their Applications in Hydrodynamics. Moscow, Nayka Publ., 1968. (in Russian)

2. Kuropatenko V.F. [On Difference Methods for Hydrodynamic Equations]. Trudy Matem. Instituta im. V.A. Steklova ANSSSR - Works MIAN, 1966, vol. 74, issue 1, pp. 107-137. (in Russian)

3. Kuropatenko V.F., Makeeva I.R. Study of Discontinuity Distraction for Methods of Shock Wave Calculations. Matem. Mod., 2006, vol. 18, no. 3, pp. 120-128. (in Russian)

4. Neumann J., Richtmayer R. A Method for the Numerical Calculation of Hydrodynamical Shocks. J. Appl. Phys, 1950, vol. 21, no. 3, pp. 232-237.

5. Lax P.D. Weak Solution of Nonlinear Hyperbolic Equations and Their Numerical Computations. Comn. Pure and Appl. Math, 1954, vol. 7, pp. 159-193.

6. Godunov S.K. Difference Method of Computation of Shock Waves. Uspekhi Mat. Nauk, 1957, no. 12, issue 1(73), pp. 176-177. (in Russian) 
7. Kuropatenko V.F. [A Shock Calculation Method]. DAN SSSR, 1960, vol. 3, no. 4, pp. 771-772. (in Russian)

8. Kuropatenko V.F. Local Conservativeness of Difference Schemes for Equations of Gas Dynamics. Zh. Vychisl. Mat. Mat. Fiz., 1985, vol. 25, no. 8, pp. 1176-1188. (in Russian)

9. Kuropatenko V.F., Dorovskikh I.A., Makeyeva I.R. The Effects of Difference Schemes Properties on the Mathematical Simulation of Dynamic Processes. Computational Technology, 2006, vol. 11, no. 2, special issue, pp. 3-12. (in Russian)

10. Kuropatenko V.F., Kovalenko G.V., Kuznetsova V.I., Mikhaylova G.I. Code and Inhomogeneous Difference Method for Simulating Non-Equilibrium Compressible Continuum Flows. Problems in Nuclear Science and Engineering Journal, Series: Mathematical Modeling of Physical Processes, 1989, issue 2, pp. 9-17. (in Russian)

Valentin F. Kuropatenko, Doctor of Physico-Mathematical Sciences, Full Professor, Russian Federal Nuclear Center E. I. Zababakhin All-Russian Scientific Research Institute of Technical Physics, Snezhinsk, Russian Federation,v.f.kuropatenko@rambler.ru

Marina N. Yakimova, Research Assistant, RRussian Federal Nuclear Center E. I. Zababakhin All-Russian Scientific Research Institute of Technical Physics, Snezhinsk, Russian Federation, yakimovamn@yandex.ru

Received April 4, 2015 